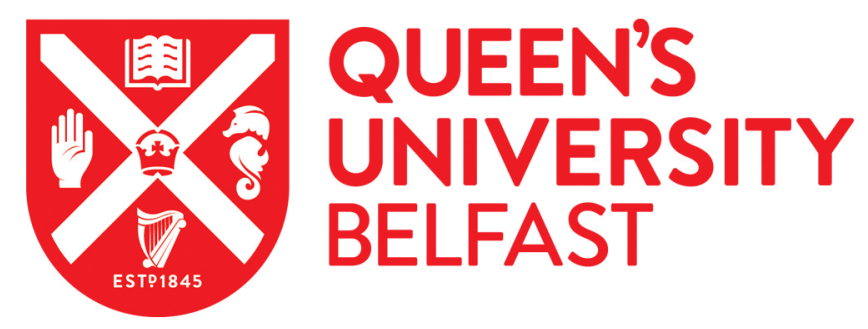

\title{
On the characteristics of obliquely propagating electrostatic structures in non-Maxwellian plasmas in the presence of ion pressure anisotropy
}

Adnan, M., Qamar, A., Mahmood, S., \& Kourakis, I. (2017). On the characteristics of obliquely propagating electrostatic structures in non-Maxwellian plasmas in the presence of ion pressure anisotropy. Physics of Plasmas, 24(3), 1-12. [032114/1-11]. https://doi.org/10.1063/1.4978613

Published in:

Physics of Plasmas

Document Version:

Publisher's PDF, also known as Version of record

Queen's University Belfast - Research Portal:

Link to publication record in Queen's University Belfast Research Portal

Publisher rights

Copyright 2017 AIP This work is made available online in accordance with the publisher's policies. Please refer to any applicable terms of use of the publisher.

\section{General rights}

Copyright for the publications made accessible via the Queen's University Belfast Research Portal is retained by the author(s) and / or other copyright owners and it is a condition of accessing these publications that users recognise and abide by the legal requirements associated with these rights.

Take down policy

The Research Portal is Queen's institutional repository that provides access to Queen's research output. Every effort has been made to ensure that content in the Research Portal does not infringe any person's rights, or applicable UK laws. If you discover content in the Research Portal that you believe breaches copyright or violates any law, please contact openaccess@qub.ac.uk. 


\section{On the characteristics of obliquely propagating electrostatic structures in non- Maxwellian plasmas in the presence of ion pressure anisotropy}

Muhammad Adnan, Anisa Qamar, Shahzad Mahmood, and loannis Kourakis

Citation: Physics of Plasmas 24, 032114 (2017);

View online: https://doi.org/10.1063/1.4978613

View Table of Contents: http://aip.scitation.org/toc/php/24/3

Published by the American Institute of Physics

\section{Articles you may be interested in}

Ion-acoustic solitary waves in a positron beam plasma with electron trapping and nonextensivity effects

Physics of Plasmas 24, 032104 (2017); 10.1063/1.4978294

Magnetosonic wave in pair-ion electron collisional plasmas

Physics of Plasmas 24, 032106 (2017); 10.1063/1.4978492

Oblique propagation of solitary electrostatic waves in magnetized plasmas with cold ions and nonthermal electrons

Physics of Plasmas 24, 022306 (2017); 10.1063/1.4976126

Formation of solitary waves and oscillatory shocklets in a two-temperature electron $\mathrm{k}$-distributed plasma Physics of Plasmas 24, 042109 (2017); 10.1063/1.4979675

Langmuir oscillations in a nonthermal nonextensive electron-positron plasma

Physics of Plasmas 24, 022116 (2017); 10.1063/1.4976128

Referee acknowledgment for 2016

Physics of Plasmas 24, 039801 (2017); 10.1063/1.4978800

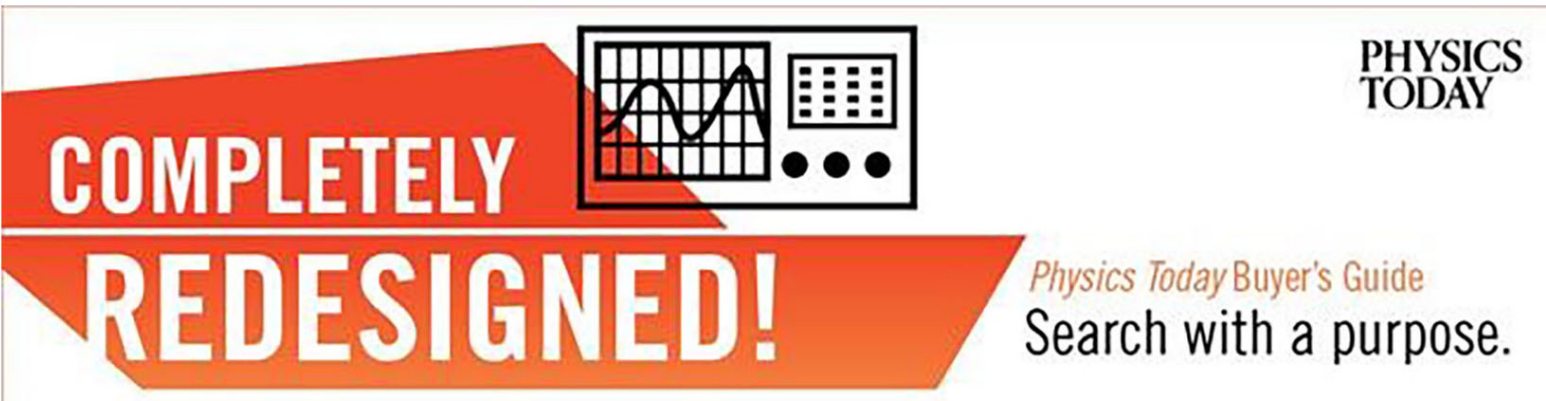




\title{
On the characteristics of obliquely propagating electrostatic structures in non-Maxwellian plasmas in the presence of ion pressure anisotropy
}

\author{
Muhammad Adnan, ${ }^{1, a)}$ Anisa Qamar, ${ }^{2}$ Shahzad Mahmood, ${ }^{3}$ and loannis Kourakis ${ }^{4}$ \\ ${ }^{1}$ Department of Physics, Kohat University of Science and Technology (KUST), Kohat, Pakistan \\ ${ }^{2}$ Department of Physics, University of Peshawar, Peshawar 25000, Pakistan \\ ${ }^{3}$ Theoretical Physics Division (TPD), PINSTECH, P.O. Nilore, Islamabad 44000, Pakistan \\ ${ }^{4}$ Department of Physics and Astronomy, Centre for Plasma Physics, Queen's University Belfast, \\ BT7 INN Northern Ireland, United Kingdom
}

(Received 30 October 2016; accepted 2 March 2017; published online 22 March 2017)

The dynamical characteristics of large amplitude ion-acoustic waves are investigated in a magnetized plasma comprising ions presenting space asymmetry in the equation of state and nonMaxwellian electrons. The anisotropic ion pressure is defined using the double adiabatic ChewGolberger-Low theory. An excess in the superthermal component of the electron population is assumed, in agreement with long-tailed (energetic electron) distribution observations in space plasmas; this is modeled via a kappa-type distribution function. Large electrostatic excitations are assumed to propagate in a direction oblique to the external magnetic field. In the linear (small amplitude) regime, two electrostatic modes are shown to exist. The properties of arbitrary amplitude (nonlinear) obliquely propagating ion-acoustic solitary excitations are thus investigated via a pseudomechanical energy balance analogy, by adopting a Sagdeev potential approach. The combined effect of the ion pressure anisotropy and excess superthermal electrons is shown to alter the parameter region where solitary waves can exist. An excess in the suprathermal particles is thus shown to be associated with solitary waves, which are narrower, faster, and of larger amplitude. Ion pressure anisotropy, on the other hand, affects the amplitude of the solitary waves, which become weaker (in strength), wider (in spatial extension), and thus slower in comparison with the cold ion case. Published by AIP Publishing. [http://dx.doi.org/10.1063/1.4978613]

\section{INTRODUCTION}

The occurrence of excess energetic electrons is a common feature in laboratory and space plasma environments in the ionosphere, the auroral zone, the mesosphere, the lower thermosphere, and elsewhere. ${ }^{1-4}$ A non-Maxwellian electron distribution, featuring an excess in the superthermal component, is often found, e.g., in Space observations, where a long tail in the energy region indicates a deviation from the Maxwellian behavior. The so-called kappa $(\kappa)$ or generalized Lorenzian velocity distribution function is often employed to model such situations. The kappa ( $\kappa)$ distribution was first postulated by Vasyliunas in $1968^{1}$ to model solar wind data and was later adopted by many researchers in various physical settings. A brief review of the different forms of kappa distribution applying to various physical scenarios can be found in Refs. 5 and 6. The application of kappa distributions includes fitting and interpretation of observations in the earth's foreshock (for $3<\kappa<6$ ) $^{7}$ and of coronal electrons in solar wind models (satisfying $2<\kappa<6) .{ }^{8,9}$ In the laboratory, a superthermal electrons are also observed in laser matter interactions or in experimental studies of plasma turbulence. ${ }^{10}$ Various theoretical investigations have by now established the theoretical background of

\footnotetext{
${ }^{a)}$ Research carried out at Department of Physics and Astronomy, Centre for Plasma Physics, Queen's University Belfast, BT7 1NN Northern Ireland, United Kingdom, while on leave from Department of Physics, University of Peshawar, Peshawar 25000, Pakistan.
}

non-Maxwellian distributions, which seem to arise as a ubiquitous characteristic of nature. ${ }^{6,11}$ This is clearly a growing area of study in plasma physics.

The three-dimensional isotropic kappa velocity distribution function reads ${ }^{5}$

$$
f_{\kappa}(v)=\frac{n_{0}}{\left(\pi \kappa \theta^{2}\right)^{3 / 2}} \frac{\Gamma(\kappa+1)}{\Gamma(\kappa-1 / 2)}\left(1+\frac{v^{2}}{\kappa \theta^{2}}\right),
$$

where $\kappa$ is the spectral index, measuring the slope of the energy spectrum of the superthermal electrons at the tail of the distribution function: lower values of $\kappa$ represent higher concentrations of superthermal electrons in the tail of the distribution function (recall that $\kappa>3 / 2$ should hold for a physically valid solution). ${ }^{5}$ In the latter formula, we have defined the Gamma function $\Gamma(x)$ and the equilibrium particle (number) density $n_{0}$. The $\kappa-$ dependent (modified) thermal speed in the presence of superthermal electrons is here denoted by $\theta=[(\kappa-3 / 2) / \kappa]^{1 / 2}\left(2 k_{B} T_{e} / m_{e}\right)^{1 / 2}$ (the Boltzmann constant is denoted by $k_{B}$ as usual). In the limit $\kappa \rightarrow \infty$, the above distribution function reduces to the Maxwellian limit, with the average electron speed being $v_{t e}=\left(2 k_{B} T_{e} / m_{e}\right)^{1 / 2}$. A comprehensive literature review on superthermal electrons can be found in Refs. 12 and 13 (also see various references cited therein).

Assuming a collisionless regime, the presence of a strong magnetic field makes the plasma anisotropic, i.e., dynamical behavior differs in the parallel and perpendicular 
direction(s), relative to the external magnetic field. ${ }^{14}$ The Chew-Goldberger-Low (CGL) theory, ${ }^{15}$ also known as the double adiabatic theory, applies to such anisotropic plasma, provided that no coupling exists between the parallel and perpendicular degrees of freedom. ${ }^{16}$ In such plasmas, one needs to separate the equations of state, i.e., to evaluate the ion pressure, viz., $p_{\|, i}$ and $p_{\perp, i}$, where $p_{\|, i}$ and $p_{\perp, i}$ denote the parallel and perpendicular, respectively, ionic pressure relative to the ambient magnetic field. In isotropic plasma, strong coupling between the parallel and perpendicular directions, due to wave-particle interaction, ${ }^{17,18}$ leads to a simplified description; hence, two separate equations of state are not necessary.

In space plasmas, there are physical regimes where magnetic compression and/or expansion processes generate plasma convection. Magnetic compression leads to an increase in the perpendicular temperature $T_{\perp}$ of the particles, while expansion is associated with a decrease in the parallel temperature $T_{\| \cdot}{ }^{17}$ These changes result in temperature anisotropy, i.e., $T_{\perp i} / T_{\| i} \neq 1$. One may find examples of stream-stream (fast and slow streams of charged particles) interactions in the solar wind, sunward flow in the terrestrial, and planetary magnetotails and magnetosheath flow around the terrestrial and planetary magnetosphere ${ }^{17}$ within the above mentioned physical situations.

Temperature anisotropy is ubiquitous in space observations in a variety of environments, such as in the Solar corona, the solar wind, the ionosphere, and the Earth's magnetosphere, with typical plasma parameters in the order of, e.g., $n \approx 10^{7}-10^{12} m^{-3}, T \approx 10^{2}-10^{7} \mathrm{~K}$ and temperature anisotropy in the range of $0.05 \leq T_{\perp} / T_{\|} \leq 24 .{ }^{17,19}$ For instance, the data for the outer Solar Corona ${ }^{19}$ obtained from the SOHO (Solar and Heliospheric Observatory) showed that the velocity distribution function is practically isotropic at about 1.8 solar radii $\left(1 R_{s}=6.9 \times 10^{8} \mathrm{~m}\right)$ and becomes anisotropic beyond that distance. Similarly, data for the solar wind $^{20,21}$ obtained from different spacecrafts, such as Helios I, Cluster II, and Ulysses, have shown the evidence of anisotropy in those regions, suggesting that the core population of the solar wind cannot be modelled via the usual Maxwellian particle distribution function. In laser-plasma interaction experiments, the incident high energy laser beam can produce a strong anisotropy in the formed plasma temperature. This is due to the fact that the formed plasma is predominantly heated in the direction of the laser wave electric field. ${ }^{22,23}$ Our work at hand is also motivated by a series of magnetosheath observations made by instruments onboard the AMPET/CCF and AMPET/IRM spacecraft missions, as described in Denton et al., ${ }^{17}$ and also independently by Seough et al. . $^{24,25}$

Ion pressure anisotropy in magnetized plasmas has been shown to bear a significant effect on the propagation characteristics of ion-acoustic solitary structures. ${ }^{26}$ The characteristics of linear modes occurring in anisotropic plasma have been studied by Gedalin ${ }^{27}$ and Gebretsadkan and Kalra ${ }^{28}$ using magnetohyrodynamics (MHD) in the framework of CGL theory. They related their results to astrophysical features such as cosmic rays and pulsar winds. Similarly, nonlinear solitary waves in magnetized plasma have been studied by Chatterjee et al. ${ }^{29}$ by adopting the Sagdeev pseudopotential approach. Considering Poisson's equation, rather than the neutrality condition (plasma hypothesis), they have obtained a set of conditions for solitary wave existence and have discussed the shape and speed of the solitary waves.

Choi et al. ${ }^{18,30,31}$ have extended earlier studies to cover obliquely propagating ion acoustic solitary waves, with respect to the ambient magnetic field. Recently, Adnan et $a .^{26}$ applied the CGL theory to study linear waves and (small-amplitude) nonlinear ion-acoustic solitary structures in non-Maxwellian plasmas, by adopting the ZakharovKuznetsov equation paradigm. The stability of such solitonic structures was also addressed in that study, in the framework of the CGL theory. In this article, we present a comprehensive study of nonlinear wave characteristics, by taking into account the combined effect of electron superthermality and ion pressure anisotropy. The Sagdeev potential methodology will be adopted, while the plasma neutrality hypothesis (plasma approximation) will be assumed to hold, for analytical tractability and simplicity. This work can be viewed as an extension of earlier work, ${ }^{18,26}$ beyond the standard isotropic plasma hypothesis.

The structure of this article goes as follows. In Section II, we present the basic model equations. In Section III, the characteristics of linear waves are briefly summarized. In Section IV, a nonlinear analytical framework for modeling large-amplitude electrostatic excitations is presented. In Sec. $\mathrm{V}$, a set of existence conditions for electrostatic pulses (solitons) is established. In Sec. VI, a parametric investigation is carried out, to trace the influence of the wave characteristics on various intrinsic plasma parameters. Finally, our results are summarized in the concluding Section VII.

\section{MODEL EQUATIONS}

We consider the propagation of electrostatic waves in a magnetized plasma, consisting of hot ions characterized by pressure anisotropy with respect to the direction of the ambient magnetic field. Particle collisions are neglected throughout this study. For the ionic pressure, we have adopted the adiabatic or Chew-Golberger-Low (CGL) description. ${ }^{15} \mathrm{~A}$ superthermal electron population is assumed to exist, associated with a presumed long-tailed distribution here modelled as a kappa distribution. The ambient magnetic field is taken to be uniform, for simplicity, and assumed to be along the $\hat{x}$ axis, i.e., $\mathbf{B}=B_{0} \hat{x}$.

The ion fluid equations in the presence of ion pressure anisotropy read

$$
\partial_{t} n_{i}+\nabla \cdot\left(n_{i} \mathbf{v}_{i}\right)=0
$$

and

$$
\partial_{t} \mathbf{v}_{i}+\left(\mathbf{v}_{i} \cdot \nabla\right) \mathbf{v}_{i}=\frac{Z e}{m_{i}} \mathbf{E}+\frac{Z e}{m_{i} c}\left(\mathbf{v}_{i} \times B_{0} \hat{x}\right)-\frac{1}{m_{i} n_{i}} \nabla \cdot \tilde{\mathbf{P}}_{\mathbf{i}} .
$$

The presence of a strong external magnetic field $B_{0}$ makes the plasma anisotropic, and hence, its behavior is different in the parallel and perpendicular direction(s). The 
pressure tensor, $\tilde{\mathbf{P}}_{\mathbf{i}}$, splits into a parallel $\left(p_{\| i}\right)$ and a perpendicular $\left(p_{\perp i}\right)$ component and takes the form ${ }^{17,26}$

$$
\tilde{\mathbf{P}}_{\mathbf{i}}=p_{\perp i} \hat{\mathbf{I}}+\left(p_{\| i}-p_{\perp i}\right) \hat{\mathbf{b}} \hat{\mathbf{b}},
$$

where $\hat{\mathbf{I}}$ is the unit tensor and $\hat{\mathbf{b}}$ is the unit vector along the external magnetic field. The parallel and perpendicular ionic pressure functions are given, respectively, by ${ }^{17,26}$

$$
p_{\| i}=p_{\| i 0}\left(\frac{n_{i}}{n_{i 0}}\right)^{3} \quad \text { and } \quad p_{\perp i}=p_{\perp i 0}\left(\frac{n_{i}}{n_{i 0}}\right),
$$

where $\mathbf{p}_{\| i 0}=\mathbf{n}_{i 0} \mathbf{k}_{B} \mathbf{T}_{i \|}$ and $\mathbf{p}_{\perp i 0}=\mathbf{n}_{i 0} \mathbf{k}_{B} \mathbf{T}_{i \perp}$ obviously represent the equilibrium values of the parallel and perpendicular pressure, respectively. In the isotropic case, the two components coincide, viz., $p_{\perp i}=p_{\| i}$; hence, the model reduces to $\nabla \cdot \tilde{\mathbf{P}}_{\mathbf{i}}=\nabla p_{i}$

For the electron number density, we have adopted the kappa distribution function

$$
n_{e}=n_{e 0}\left[1-\frac{e \phi}{k_{B} T_{e}(\kappa-3 / 2)}\right]^{-\kappa+1 / 2} .
$$

It is important to mention here that the formulation of the kappa distribution in the presence of a potential energy may incorporate $\phi-\langle\phi\rangle$ (rather than $\phi$ ), as described in Ref. 32. Importantly, in our case $\langle\phi\rangle / k_{B} T_{e} \ll 1$ (as, in fact, $\langle\phi\rangle=0$ ); hence, Eq. (5) holds.

In Eq. (5), $\kappa$ is the spectral index, measuring the slope of the energy spectrum of the electrons at the tail of the distribution function. It suffices to retain, qualitatively speaking, that the smaller the value of $\kappa$ the stronger the superthermal (energetic) electron concentration in the superthermal region of the distribution function. One recovers the Maxwellian limit upon setting $\kappa \rightarrow \infty$.

We assume that the spatial variation in the electrostatic potential is slow and essentially occurs on a scale beyond the Debye sphere, i.e., $k^{2} \lambda_{D, e}^{2} \ll 1$, where $\lambda_{D e}=\left(k_{B} T_{e}\right)$ $\left.4 \pi n_{e 0} e^{2}\right)^{1 / 2}$ is the (electron) Debye radius. Accordingly, we have adopted the plasma approximation (charge neutrality hypothesis) in our model, by closing the algebraic system of fluid equations by setting $n_{i} \simeq n_{e}$ (at all times). Obviously, charge neutrality at equilibrium requires $n_{i 0}=n_{e 0}$.

\section{A. Fluid evolution equations}

For simplicity, we have assumed that any excitation of equilibrium evolves and propagates in the $x y$-plane only, viz., $\partial / \partial z=0$, with no loss of generality. The above system of equation thus takes the form

$$
\begin{gathered}
\partial_{t} n_{i}+\partial_{x}\left(n_{i} v_{i x}\right)+\partial_{y}\left(n_{i} v_{i y}\right)=0 \\
\partial_{t} v_{i x}+\left(v_{i x} \partial_{x}+v_{i y} \partial_{y}\right) v_{i x}=-\frac{e}{m_{i}} \partial_{x} \phi-\frac{3 p_{\| i 0}}{m_{i} n_{0}^{3}} n_{i} \partial_{x} n_{i} \\
\partial_{t} v_{i y}+\left(v_{i x} \partial_{x}+v_{i y} \partial_{y}\right) v_{i y}=-\frac{e}{m_{i}} \partial_{y} \phi+\Omega_{i} v_{i z}-\frac{p_{\perp i 0}}{m_{i} n_{0} n_{i}} \partial_{y} n_{i},
\end{gathered}
$$

$$
\partial_{t} v_{i z}+\left(v_{i x} \partial_{x}+v_{i y} \partial_{y}\right) v_{i z}=-\Omega_{i} v_{i y},
$$

and

$$
n_{i} \simeq n_{e}=n_{e 0}\left[1-\frac{e \phi}{k_{B} T_{e}(\kappa-3 / 2)}\right]^{-\kappa+1 / 2} .
$$

Here, the quantities $e, m_{i}, n_{e}, n_{i}$, and $\phi$ represent the electronic charge, ion mass, electron fluid density, ion fluid density, and electrostatic potential, respectively. The ion gyrofrequency is defined by $\Omega_{i}=\frac{e B_{0}}{m_{i} c}$ while $v_{i x}, v_{i y}$, and $v_{i z}$ denote the fluid velocity components. Note that the ionic charge state has been set equal to unity, i.e., $Z_{i}=1$, everywhere.

\section{B. Scaling}

We shall rely in the following on a normalized form of the above equations

$$
\begin{gathered}
\partial_{t} n_{i}+\partial_{x}\left(n_{i} v_{i x}\right)+\partial_{y}\left(n_{i} v_{i y}\right)=0, \\
\partial_{t} v_{i x}+\left(v_{i x} \partial_{x}+v_{i y} \partial_{y}\right) v_{i x}=-\partial_{x} \Phi-P_{\|} n_{i} \partial_{x} n_{i}, \\
\partial_{t} v_{i y}+\left(v_{i x} \partial_{x}+v_{i y} \partial_{y}\right) v_{i y}=-\partial_{y} \Phi+\Omega v_{i z}-\frac{P_{\perp}}{n_{i}} \partial_{y} n_{i}, \\
\partial_{t} v_{i z}+\left(v_{i x} \partial_{x}+v_{i y} \partial_{y}\right) v_{i z}=-\Omega v_{i y}
\end{gathered}
$$

and

$$
n_{i} \simeq n_{e}=\left[1-\frac{\Phi}{(\kappa-3 / 2)}\right]^{-\kappa+1 / 2} .
$$

In the latter system of equations, the number density variables $n_{j}$ (for species $j=e, i$ ) have been scaled by the equilibrium ion density $n_{i 0}$, the electrostatic potential $\phi$ by $\left(T_{e} / e\right)$, while the ion fluid speed (vector components) has been normalized by the ion sound speed $c_{s}=\left(T_{e} / m_{i}\right)^{1 / 2}$. The space and time variables are normalized by the electron Debye radius $\lambda_{D e}=\left(k_{B} T_{e} / 4 \pi n_{e} 0 e^{2}\right)^{1 / 2}$ and the inverse ion plasma frequency $\omega_{p i}^{-1}=\left(m_{i} / 4 \pi n_{i 0} e^{2}\right)^{1 / 2}$, respectively. In the above relations, the dimensionless parameter $\Omega$ denotes the ratio $\Omega=\frac{\Omega_{i}}{\omega_{p i}}$, while $P_{\|}=\frac{3 p_{\| i 0}}{n_{i 0} T_{e}}$ and $P_{\perp}=\frac{p_{\perp i 0}}{n_{i 0} T_{e}}$ express the relative strength of the pressure, normalized by the thermal pressure in the respective directions.

\section{LINEAR DISPERSION RELATION}

One may assume small-amplitude harmonic perturbations in the form $\sim e^{i\left(k_{x} x+k_{y} y-\omega t\right)}$, i.e., analyzing Eqs. (11)-(15) by Fourier. The wavenumbers in the directions parallel (perpendicular) to the magnetic field are, respectively, denoted by $k_{x}\left(k_{y}\right)$, so that $k_{x}^{2}+k_{y}^{2}=k^{2}$. One thus obtains a dispersion relation (DR) linear wave propagation in the form

$$
1=\left(\frac{\kappa-3 / 2}{\kappa-1 / 2}+P_{\|}\right) \frac{k_{x}^{2}}{\omega^{2}}+\left(\frac{\kappa-3 / 2}{\kappa-1 / 2}+P_{\perp}\right) \frac{k_{y}^{2}}{\omega^{2}-\Omega^{2}} .
$$

One notices immediately the explicit dependence of ion pressure anisotropy on the DR, via $P_{\|}$and $P_{\perp}$ (recall that these were defined above as $P_{\|}=\frac{3 p_{\| i 0}}{n_{i 0} k_{B} T_{e}}$ and $P_{\perp}=\frac{p_{\perp i 0}}{n_{i 0} k_{B} T_{e}}$, 
respectively). Furthermore, the magnetic field appears through the (cyclotron- to plasma-) frequency ratio $\Omega$, while the effect of electron superthermality enters into play through $\kappa$. Equation (16) can be expressed as

$$
\omega_{ \pm}^{2}=\frac{1}{2}\left[\left(\frac{\kappa-3 / 2}{\kappa-1 / 2} k^{2}+P_{\|} k_{x}^{2}+P_{\perp} k_{y}^{2}+\Omega^{2}\right) \pm \sqrt{\left(\frac{\kappa-3 / 2}{\kappa-1 / 2} k^{2}+P_{\|} k_{x}^{2}+P_{\perp} k_{y}^{2}+\Omega^{2}\right)^{2}-4\left(\frac{\kappa-3 / 2}{\kappa-1 / 2}+P_{\|}\right) \Omega^{2} k_{x}^{2}}\right]
$$

suggesting that two branches occur, $\omega_{+}$and $\omega_{-}$, corresponding to a fast and a slow electrostatic mode, respectively. In the following, we shall discuss some of the resulting limiting cases and their properties, for later reference.

\section{A. Parallel propagation}

The dispersion relation for parallel-propagating electrostatic waves in a warm magnetized plasma with superthermal electrons can be obtained by setting, $k_{y} \rightarrow 0$ (thus $k_{x}=k$ ) in Equation (17), as

$$
\omega_{-}^{2}(k)=\left(\frac{\kappa-3 / 2}{\kappa-1 / 2}+P_{\|}\right) k^{2}
$$

Note that neither the magnetic field strength $\Omega$ nor the ion perpendicular pressure $P_{\perp}$ contribute to the above relation. The phase speed (essentially, the sound speed) in the direction parallel to the magnetic field is thus readily obtained as

$$
v_{p h}=\left(\frac{\kappa-3 / 2}{\kappa-1 / 2}+P_{\|}\right)^{1 / 2}
$$

\section{LARGE ELECTROSTATIC EXCITATIONS}

In this section, we shall investigate the existence of large (arbitrary amplitude) solitary waves, in the presence of ion pressure anisotropy, in non-Maxwellian plasmas. In order to obtain a solitary wave solution for Equations (11)-(15), we introduce a moving variable $\xi=\alpha x+\beta y-M t$, where $M=\frac{V}{c_{s}}$ is the normalized pulse velocity (with $V$ denoting the soliton speed). The parameters $\alpha$ and $\beta$ here denote the directional cosines along the $x$ and $y$ directions, i.e., $\alpha=k_{x} / k$ $=\cos \theta$ and $\beta=k_{y} / k=\sin \theta$ (viz., $\alpha^{2}+\beta^{2}=1$.)

Assuming that all fluid variables in evolution Equations (11)-(15) depend on $\xi$, one is led to a set of coupled ordinary differential equations in the co-moving co-ordinate $\xi$. The transformed equations read

$$
\begin{gathered}
-M \frac{d n_{i}}{d \xi}+\alpha \frac{d\left(n_{i} v_{i x}\right)}{d \xi}+\beta \frac{d\left(n_{i} v_{i y}\right)}{d \xi}=0 \\
\left(-M+\alpha v_{i x}+\beta v_{i y}\right) \frac{d v_{i x}}{d \xi}+\alpha \frac{d \Phi}{d \xi}+\alpha P_{\|} n_{i} \frac{d n_{i}}{d \xi}=0 \\
\left(-M+\alpha v_{i x}+\beta v_{i y}\right) \frac{d v_{i y}}{d \xi}+\beta \frac{d \Phi}{d \xi}-\Omega v_{i z}+\beta P_{\perp} \frac{1}{n_{i}} \frac{d n_{i}}{d \xi}=0
\end{gathered}
$$

$$
\left(-M+\alpha v_{i x}+\beta v_{i y}\right) \frac{d v_{i z}}{d \xi}+\Omega v_{i y}=0
$$

Integrating the above set of Eqs. (20)-(23) with appropriate boundary conditions, i.e., $n_{i} \rightarrow 1, v_{i x, y} \rightarrow 0$ and $\Phi \rightarrow$ 0 , at $\xi \rightarrow \pm \infty$, and adopting the neutrality approximation, viz., $n_{i} \simeq n_{e}=n$, we obtain

$$
\begin{gathered}
\left(\alpha v_{x}+\beta v_{y}\right)=M\left(\frac{n-1}{n}\right), \\
v_{x}=\frac{\alpha}{M}\left\{-1+\int n d \Phi+\frac{1}{3} P_{\|}\left(n^{3}-1\right)\right\}, \\
v_{y}=\frac{M}{\beta}\left(1-\frac{1}{n}\right)-\frac{\alpha^{2}}{M \beta}\left\{-1+\int n d \Phi+\frac{1}{3} P_{\|}\left(n^{3}-1\right)\right\} .
\end{gathered}
$$

For simplicity, we have dropped the subscript $i$ in writing down the above equations. After a lengthy algebraic manipulation, one can obtain the following relation: ${ }^{33-35}$

$$
\begin{aligned}
\frac{d^{2} S}{d \xi^{2}}= & \frac{d^{2}}{d \xi^{2}}\left[\Phi+\frac{M^{2}}{2}\left(1-\frac{\Phi}{\kappa-3 / 2}\right)^{2 \kappa-1}\right. \\
& +\frac{\alpha^{2} P_{\|}}{2}\left(1-\frac{\Phi}{\kappa-3 / 2}\right)^{-2 \kappa+1} \\
& \left.+\beta^{2} P_{\perp} \ln \left(1-\frac{\Phi}{\kappa-3 / 2}\right)^{-\kappa+1 / 2}\right]=F(\Phi),
\end{aligned}
$$

where the function $S$ represents the quantity in the square brackets, and $F(\Phi)$ is given by

$$
\begin{aligned}
F(\Phi)= & \Omega^{2}\left[\left(1+\frac{\alpha^{2}}{M^{2}}\right)\left(1-\frac{\Phi}{\kappa-3 / 2}\right)^{-\kappa+1 / 2}-1\right. \\
& -\frac{\alpha^{2}}{M^{2}}\left(1-\frac{\Phi}{\kappa-3 / 2}\right)^{-2 \kappa+2} \\
& +\frac{\alpha^{2}}{3 M^{2}} P_{\|}\left(1-\frac{\Phi}{\kappa-3 / 2}\right)^{-\kappa+1 / 2} \\
& \left.\times\left\{1-\left(1-\frac{\Phi}{\kappa-3 / 2}\right)^{-3 \kappa+3 / 2}\right\}\right]
\end{aligned}
$$

Multiplying both sides of Equation (27) by $d S / d \xi$ and integrating once, we obtain a pseudo-energy-conservation condition in the form 


$$
\frac{1}{2}\left(\frac{d \Phi}{d \xi}\right)^{2}+\Psi\left(\Phi, M, \kappa, P_{\|}, P_{\perp}\right)=0,
$$

where

$$
\Psi\left(\Phi, M, \kappa, P_{\|}, P_{\perp}\right)=\Omega^{2} \frac{\Psi_{1}\left(\Phi, M, \kappa, P_{\|}, P_{\perp}\right)}{\Psi_{2}\left(\Phi, M, \kappa, P_{\|}, P_{\perp}\right)}
$$

is a "pseudopotential" function, with $\Psi_{1}\left(\Phi, M, \kappa, P_{\|}, P_{\perp}\right)$ and $\Psi_{2}\left(\Phi, M, \kappa, P_{\|}, P_{\perp}\right)$ given by

$$
\begin{aligned}
& \Psi_{1}\left(\Phi, M, \kappa, P_{\|}, P_{\perp}\right)=\left(1+\frac{\alpha^{2}}{M^{2}}\right)\left[1-\left(1-\frac{\Phi}{\kappa-3 / 2}\right)^{-\kappa+3 / 2}\right]-\frac{M^{2}}{2}\left[1-\left(1-\frac{\Phi}{\kappa-3 / 2}\right)^{2 \kappa-1}\right] \\
& +\left[1-\alpha^{2}\left(\frac{\kappa-1 / 2}{\kappa-3 / 2}\right) \Phi\right]+\left(M^{2}+\alpha^{2}\right)\left[1-\left(1-\frac{\Phi}{\kappa-3 / 2}\right)^{\kappa-1 / 2}\right]-\frac{\alpha^{2}}{2 M^{2}}\left[1-\left(1-\frac{\Phi}{\kappa-3 / 2}\right)^{-2 \kappa+3}\right] \\
& +P_{\|}\left\{\frac{\alpha^{2}}{3 M^{2}}\left[1-\left(1-\frac{\Phi}{\kappa-3 / 2}\right)^{-\kappa+3 / 2}\right]-\frac{\alpha^{2}}{12 M^{2}}\left(\frac{\kappa-3 / 2}{\kappa-3 / 4}\right)\left[1-\left(1-\frac{\Phi}{\kappa-3 / 2}\right)^{-4 \kappa+3}\right]\right. \\
& -\frac{\alpha^{2}}{3}\left(1-\frac{\Phi}{\kappa-3 / 2}\right)^{\kappa-1 / 2}\left[1-\left(1-\frac{\Phi}{\kappa-3 / 2}\right)^{-3 \kappa+3 / 2}\right]+\left(1+\frac{\alpha^{2}}{M^{2}}\right) \frac{\alpha^{2}}{3}\left[1-\left(1-\frac{\Phi}{\kappa-3 / 2}\right)^{-3 \kappa+3 / 2}\right] \\
& -\frac{\alpha^{4}}{4 M^{2}}\left(\frac{\kappa-1 / 2}{\kappa-3 / 4}\right)\left[1-\left(1-\frac{\Phi}{\kappa-3 / 2}\right)^{-4 \kappa+3}\right]+\frac{\alpha^{4} P_{\|}}{9 M^{2}}\left[1-\left(1-\frac{\Phi}{\kappa-3 / 2}\right)^{-3 \kappa+3 / 2}\right] \\
& \left.-\frac{\alpha^{4} P_{\|}}{18 M^{2}}\left[1-\left(1-\frac{\Phi}{\kappa-3 / 2}\right)^{-6 \kappa+3}\right]\right\}+P_{\perp}\left\{\left(1+\frac{\alpha^{2}}{M^{2}}\right) \beta^{2}\left[1-\left(1-\frac{\Phi}{\kappa-3 / 2}\right)^{-\kappa+1 / 2}\right]\right. \\
& \left.-\beta^{2} \log \left(1-\frac{\Phi}{\kappa-3 / 2}\right)^{\kappa-1 / 2}-\frac{\alpha^{2} \beta^{2}}{2 M^{2}}\left(\frac{\kappa-1 / 2}{\kappa-1}\right)\left[1-\left(1-\frac{\Phi}{\kappa-3 / 2}\right)^{-2 \kappa+2}\right]\right\} \\
& -\frac{\alpha^{2} \beta^{2} P_{\|} P_{\perp}}{3 M^{2}}\left\{\left[1-\left(1-\frac{\Phi}{\kappa-3 / 2}\right)^{-\kappa+1 / 2}\right]-\frac{1}{4}\left[1-\left(1-\frac{\Phi}{\kappa-3 / 2}\right)^{-4 \kappa+2}\right]\right\}
\end{aligned}
$$

and

$$
\begin{aligned}
\Psi_{2}\left(\Phi, M, \kappa, P_{\|}, P_{\perp}\right)= & {\left[1-M^{2}\left(\frac{\kappa-1 / 2}{\kappa-3 / 2}\right)\left(1-\frac{\Phi}{\kappa-3 / 2}\right)^{2 \kappa-2}+P_{\|} \alpha^{2}\left(\frac{\kappa-1 / 2}{\kappa-3 / 2}\right)\left(1-\frac{\Phi}{\kappa-3 / 2}\right)^{-2 \kappa}\right.} \\
& \left.+P_{\perp} \beta^{2}\left(\frac{\kappa-1 / 2}{\kappa-3 / 2}\right)\left(1-\frac{\Phi}{\kappa-3 / 2}\right)^{-1}\right]^{2} .
\end{aligned}
$$

The main steps of the tedious derivation of Equation (29) are provided in the Appendix.

Equation (29) admits solitary wave solutions, provided that the following requirements are fulfilled:

1. The pseudopotential function $\Psi\left(\Phi, M, \kappa, P_{\|}, P_{\perp}\right)$ attains a maximum value at the origin, viz., $\Psi(\Phi=0)=0,\left.\frac{d \Psi}{d \xi}\right|_{\Phi=0}$ and $\left.\frac{d^{2} \Psi}{d \xi^{2}}\right|_{\Phi=0}<0$

2. A (one, at least) nonzero root occurs, e.g., at $\Phi=\Phi_{m}$ (here the subscript " $m$ " may either stand for " $m i n$ " or for "max"), so that $\Psi\left(\Phi_{m}, M\right)=0$ holds.

The sign of the root $\left(\Phi_{m}\right)$ determines the polarity of the potential pulse: if $\Phi_{m}<0$, then $\Psi(\Phi)$ is negative in the interval $0<\Phi<\Phi_{m}$; similarly, if $\Phi_{m}>0$, then $\Psi(\Phi)$ is positive in the interval $\Phi_{m}<\Phi<0$.
In the following, we shall demonstrate the above conditions, analytically and graphically.

\section{SOLITON EXISTENCE CONDITIONS}

In this section, we will discuss the properties of the Sagdeev-type pseudopotential $\Psi\left(\Phi, M, \kappa, P_{\|}, P_{\perp}\right)$, in order to determine the existence domain for localized nonlinear structures to occur, in terms of the various relevant parameters.

The local maximum of the function $\Psi\left(\Phi, M, \kappa, P_{\|}, P_{\perp}\right)$ at the origin $(\Phi=0)$ defines the equilibrium state. It is straightforward to show that the conditions $\Psi(\Phi=0)$ $=\left.\frac{d \Psi}{d \xi}\right|_{\Phi=0}=0$ hold, based on Eq. (30). Investigating the condition $\left.\frac{d^{2} \Psi}{d \xi^{2}}\right|_{\Phi=0}<0$, one defines a region of velocity values 
where nonlinear excitations may exist. ${ }^{38}$ Adopting the methodology presented in Refs. 34 and 35, the latter condition takes the form of the requirement

$$
\left.\frac{d^{2} \Psi}{d \xi^{2}}\right|_{\Phi=0}=\Omega^{2} \frac{M^{2}-M_{1}^{2}}{M^{2}\left(M^{2}-M_{2}^{2}\right)}<0
$$

with

$$
M_{1}=|\alpha|\left(\frac{\kappa-3 / 2}{\kappa-1 / 2}+P_{\|}\right)^{1 / 2} \leq 1
$$

and

$$
M_{2}=\left(\frac{\kappa-3 / 2}{\kappa-1 / 2}+\alpha^{2} P_{\|}+\left(1-\alpha^{2}\right) P_{\perp}\right)^{1 / 2} .
$$

It is obvious from Eq. (32) that the lower Mach number (threshold) $M_{1}$ is independent from the perpendicular pressure $P_{\perp}$, while the upper Mach number $M_{2}$ on the other hand depends on both $P_{\|}$and $P_{\perp}$. Notice that, upon setting $\alpha=1$ (on account of parallel propagation), both expressions reduce to the phase speed obtained in (19) above.

Equation (31) is satisfied for Mach number values in the interval

$$
M_{1}<M<M_{2}
$$

i.e.,

$$
\alpha<\frac{M}{M_{2}}<1
$$

with straightforward interpretation, recalling that $\alpha=$ $\cos \theta \leq 1$ is defined as a directional cosine. As expected, the expressions for $M_{1}$ and $M_{2}$ recover precisely the results of Refs. 34 and 35 in the limit(s) of cold ion plasma and isotropic plasma, respectively. It is also important to point out here, for rigor, that our results are valid in the long wavelength limit (since the neutrality hypothesis was adopted, rather than Poisson's equation), and therefore, one cannot recover the results by Saini et al. ${ }^{36}$ in unmagnetized superthermal electron-ion plasma.

A qualitative discussion is in row at this point, for the sake of rigor. First, let us recall that soliton-like pulses predicted via the pseudopotential method ${ }^{38}$ are always superacoustic (supersonic), i.e., they propagate at a speed that exceeds the true acoustic speed (for a given plasma configuration), ${ }^{39}$ namely, here given by Eq. (19). This is a (physically expected) algebraic consequence of the curvature requirement (at the origin), discussed above. ${ }^{39}$ Upon inspection of Eq. (32), one might be tempted to argue that the "supersonic soliton" requirement is violated in our case. However, recalling that we are here focusing on oblique pulse propagation (with respect to the direction of the magnetic field), it turns out that the supersonic requirement is precisely expressed as $M>M_{1}$ [cf. Eq. (32)], since $M_{1}$ essentially accounts for the sound speed projection (vector component) in the direction of the magnetic field. Furthermore, it is stressed that this model is valid only for oblique propagation, strictly speaking. In other words, either taking $\alpha=1$, on account of parallel propagation, or setting $\Omega=0$, one does not recover the known Sagdeev-theory based results in the unmagnetized case. The reason for this is that the neutrality hypothesis was adopted herewith, which cannot be satisfied (i.e., is violated) in the parallel propagation model. These apparent contradictions have also briefly been discussed in Ref. 34 .

In order to gain some physical intuition in the problem, we have depicted the existence region for solitary waves, that is, the area bounded by the lower and upper Mach number limits, $M_{1}$ and $M_{2}$, defined above. In Figure 1(a), the Mach number range is depicted versus the superthermality parameter $\kappa$, for different values of (the obliqueness cosine) $\alpha$, in the
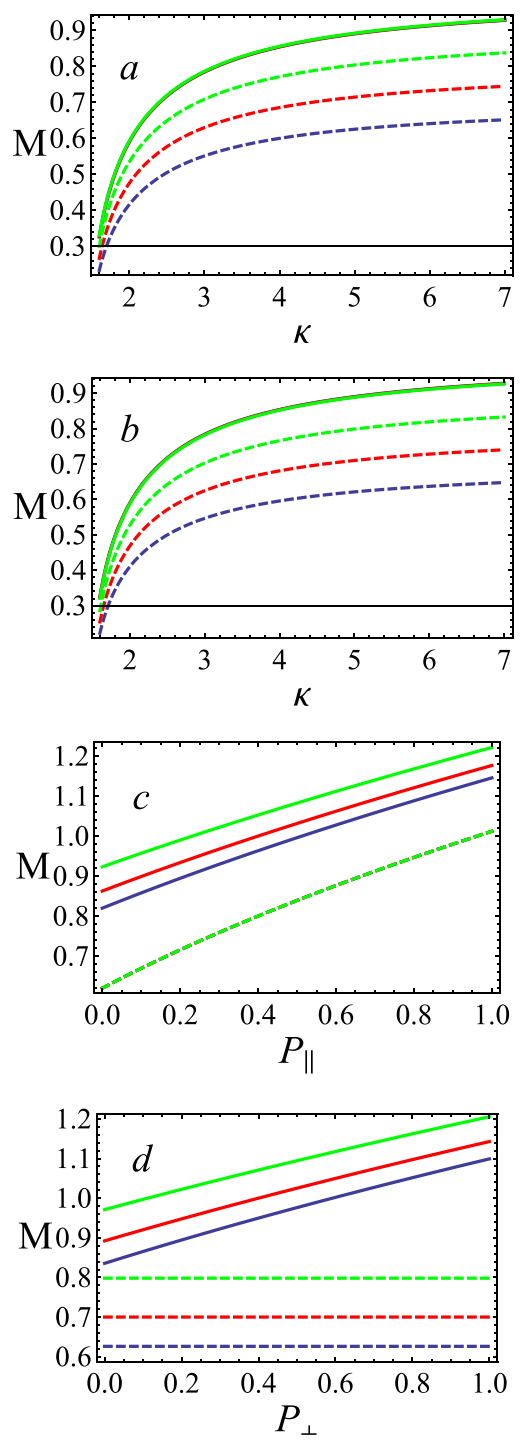

FIG. 1. The critical Mach number values $M_{1}$ (dashed curves, on top) and $M_{2}$ (solid curves, at the bottom) are depicted against relevant plasma parameters, namely (top to bottom), (a) the superthermality index $\kappa$ (taking $P_{\|}$ $=0.01$ and $P_{\perp}=0.02$, viz., $P_{\|}<P_{\perp}$, and $\alpha=0.7,0.8,0.9$ : blue, red, and green curves, respectively); (b) the superthermality index $\kappa$, with $P_{\|}$ $=0.02, P_{\perp}=0.01$ (i.e., $P_{\|}>P_{\perp}$ ) and $\alpha=0.7,0.8,0.9$ (blue, red, and green curves, respectively); (c) the parallel pressure $P_{\|}$, with $\alpha=0.8, \kappa=3$, and $P_{\perp}=0.2,0.4,0.7$ (blue, red, and green curves, respectively); (d) the perpendicular pressure $P_{\perp}$, with $\alpha=0.7, \kappa=3$, and $P_{\|}=0.2,0.4,0.7$ (blue, red, and green curves, respectively). 
case when ion parallel pressure is greater than the ion perpendicular pressure. One can see that both critical values $\left(M_{1}\right.$ and $M_{2}$ ) increase with $\kappa$, and in fact attain a constant value for high $\kappa$, as expected (recalling that large $\kappa$ essentially accounts for Maxwellian electrons). These asymptotic values clearly depend on the considered value of $\alpha$. It is evident on the graphs that the lower Mach number (threshold) $M_{1}$ increases with $\alpha$, while the upper limit $M_{2}$ is practically independent from $\alpha$. Therefore, the soliton existence range shrinks with larger deviation from the magnetic field direction, while at the same time it shifts towards higher values. Similar results are obtained and depicted in Figure 1(b), in the case when the perpendicular ion pressure is greater than the parallel ion pressure. In Fig. 1(c), we have plotted the Mach number region against the parallel pressure $P_{\|}$with different values of the perpendicular ion pressure $P_{\perp}$. One sees that the values of both lower and upper Mach numbers increase with the parallel ion pressure for a specific value of $P_{\perp}$. Increasing the perpendicular pressure essentially leads to an increase in $M_{2}$. On the other hand, $M_{1}$ is not on $P_{\perp}$, as evident from Equation (32). In an analogous manner, we have plotted $M_{1,2}$ against $P_{\perp}$ under the effect of the parallel ion pressure in Figure 1(d). Here, both the upper and lower Mach numbers (critical values) vary with $P_{\|}$; however, since the lowest Mach number is independent from the ion perpendicular pressure, the curve representing $M_{1}$ (Mach number threshold) versus $P_{\perp}$ is horizontal, that is, constant (regardless of the value of $P_{\perp}$ ).

\section{PARAMETRIC INVESTIGATION}

In this section, we shall investigate the dependence of the pseudopotential function $\Psi(\Phi)$ on various relevant plasma parameters $\left(M, \kappa, P_{\|}, P_{\perp}, \alpha, \Omega\right)$.

\section{A. Effect of superthermality}

The effect of superthermal electrons on the propagation characteristics of nonlinear ion acoustic waves has been studied by Saini et l. $^{36}$ and by Sultana et al. ${ }^{34}$ for unmagnetized and magnetized plasma, respectively. It has been shown that smaller values of the superthermality parameter $\kappa$ (i.e., larger deviation from Maxwellian equilibrium) enhances solitary waves. Recently, Singh et al. $^{35}$ confirmed the same qualitative result in the warm ion fluid model. Elaborating on those earlier works, we have investigated the effect of superthermality on the propagation of ion acoustic waves in the presence of ion temperature anisotropy. Our results are in agreement with all known previous works ${ }^{34-36}$ in the appropriate limiting cases. We observe that lower values of $\kappa$ lead to an increase in the amplitude of the soliton, which is more localized in space and also steeper, as shown in Figure 2. Considering weak obliqueness with respect to the direction of the magnetic field (in view of the electrostatic approximation ${ }^{37}$ ), and tracing the effect of superthermality on the wave dynamics, one notices that the allowed interval is wider, as $P_{\perp}$ varies, whereas $P_{\|}$determines a shorter range of values satisfying the boundary conditions; the existence region is therefore less sensitive to changes in the parallel pressure component $P_{\|}$.
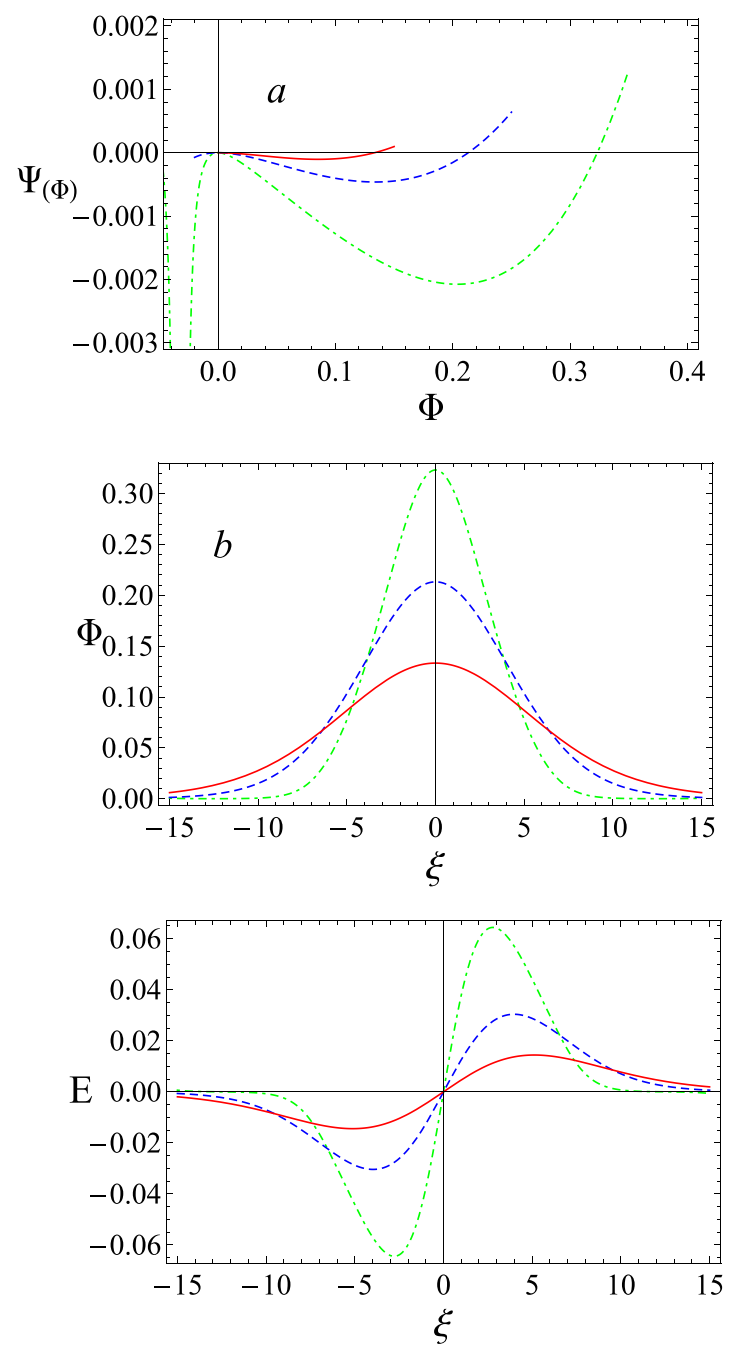

FIG. 2. (a) The pseudopotential $\Psi(\Phi)$ is plotted versus $\Phi$ for $M=$ $0.75, P_{\|}=0.01, P_{\perp}=0.02, \alpha=0.8, \Omega=0.5$ and $\kappa=3$ for dotted-dashed (green) curve; $\kappa=4$ for dashed (blue) curve; $\kappa=5$ for solid (red) curve. (b) The corresponding electrostatic potential (pulse) and (c) the resulting electric field are depicted, for the same values as in the upper frame.

\section{B. Effect of Mach number}

In Figure 3, we have depicted the Sagdeev potential form and the resulting electrostatic potential and electric field (disturbances), for various values of the Mach number $M$, keeping the remaining plasma parameters fixed. One sees in Figure 3 that, as $M$ increases, the amplitude and depth of the pseudopotential increase well; hence, structures with large $M$ value (within the region $M_{1}<M<M_{2}$ ) are taller and sharper. This is in agreement with Ref. 34. Upon simple comparison with Fig. 7 in Ref. 34, one notices a similar behavior, although the predicted pulses (solitons) are now smaller in both amplitude and width: This is due to ion pressure (anisotropy) effect.

\section{Effect of magnetic field}

The effect of the magnetic field on the characteristics of solitary waves can be traced through the (cyclotron- to plasma-) frequency ratio $\Omega$. It is quite evident from Figure 4 that the amplitude of the soliton is independent from $\Omega$, 

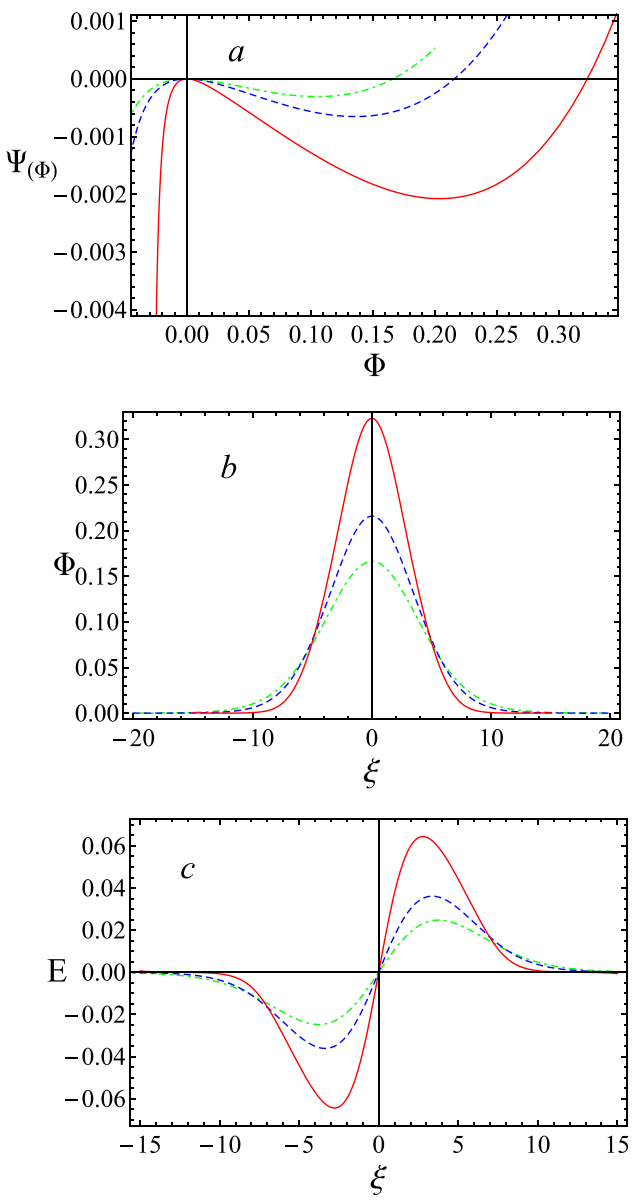

FIG. 3. (a) The pseudopotential $\Psi(\Phi)$ is plotted versus $\Phi$ for $\kappa=3, P_{\|}=$ $0.01, P_{\perp}=0.02, \alpha=0.8, \Omega=0.5$ and $M=0.68$ for dotted-dashed (green) curve; $M=0.7$ for dashed (blue) curve; $M=0.75$ for solid (red) curve. (b) The corresponding electrostatic potential (pulse) and (c) the resulting electric field are depicted, for the same values as in the upper frame.

although the depth of the Sagdeev pseudopotential (but not its root) increases well with stronger magnetic field (larger $\Omega$ ). This means that solitons in stronger magnetic fields are steeper and sharper in form. Another important aspect to recall here is that the soliton existence region (velocity range), as discussed in Section VIC, is essentially independent of $\Omega$.

\section{Effect of obliqueness}

We have also investigated the effect of the propagation direction (via the angle cosine $\alpha$ ) on the ion acoustic wave characteristics. It is important to keep the angle small (i.e., the cosine value $\alpha=\cos \theta$ large), in order to preserve the validity of the electrostatic approximation, as argued by Verheest. ${ }^{37}$ We found that, for a fixed set of plasma (configurational) parameter and Mach number $M$ values, increasing the value of $\alpha$ leads to a decrease in the soliton amplitude and also in the (depth and root of) the Sagdeev potential form, as shown in Figure 5. This is an expected qualitative result, since the Mach number threshold $M_{1}$ decreases for larger obliqueness (cf. Figure 1); hence, a given $M$ value exceeds $M_{1}$ even further and hence leads to an increased (as "more supersonic") pulse amplitude.
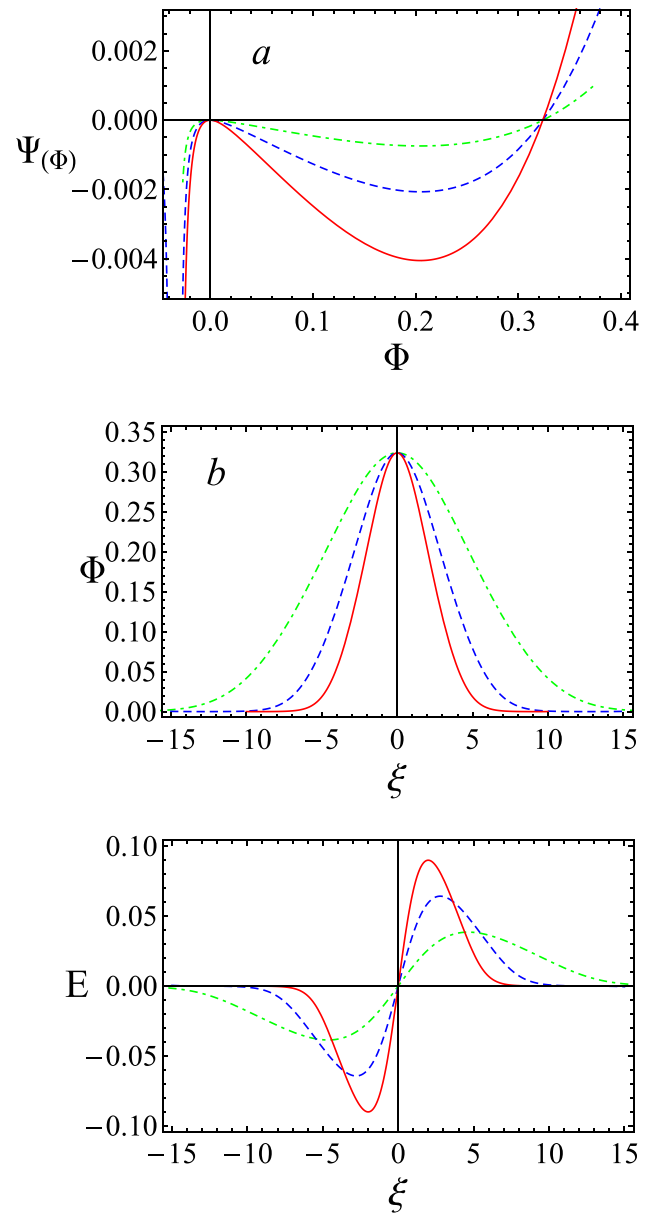

FIG. 4. (a) The pseudopotential $\Psi(\Phi)$ is plotted versus $\Phi$ for $\kappa=3, M=0.75, P_{\|}=0.01, P_{\perp}=0.03, \alpha=0.8$, and $\Omega=0.3$ for dotteddashed (green) curve; $\Omega=0.5$ for dashed (blue) curve; $\Omega=0.7$ for solid (red) curve. (b) The corresponding electrostatic potential (pulse) and (c) the resulting electric field are depicted, for the same values as in the upper frame.

\section{E. Effect of ion pressure anisotropy}

To demonstrate the effect of pressure anisotropy on the solitary waves, we have drawn the Sagdeev pseudopotential along with the corresponding electrostatic electric field perturbations, for fixed values of the plasma parameters, such that $\kappa=3, M=0.7, \alpha=0.7, P_{\perp}=0.01$, and $P_{\|}=0.03,0.06,0.09$ denoting the green, blue, and red curves, respectively, in Figure 6. We have found that, upon assuming larger values of $\alpha$ (i.e., 0.7, 0.8, and 0.9), the effect of ion pressure $P_{\|}$is more effective (i.e., a small change in $P_{\|}$leads to larger changes in $\Psi(\Phi)$ ); an increase in the parallel pressure $P_{\|}$thus leads to a decrease in the pulse (soliton) amplitude, as shown in Figure 6. A similar result is obtained for the perpendicular ion pressure. Similarly, in Figure 7, we have considered three different cases, namely, $P_{\|}>P_{\perp}, P_{\|}<P_{\perp}$ and $P_{\|}=P_{\perp}=0$. We see that the characteristics of ion acoustic pulses are more sensitive to variations of the parallel ion pressure $\left(P_{\|}\right)$ rather than its perpendicular counterpart $\left(P_{\perp}\right)$. Another important feature of Figure 7 is that, in general, the ion thermal pressure reduces both the amplitude and the width of electrostatic pulses. 

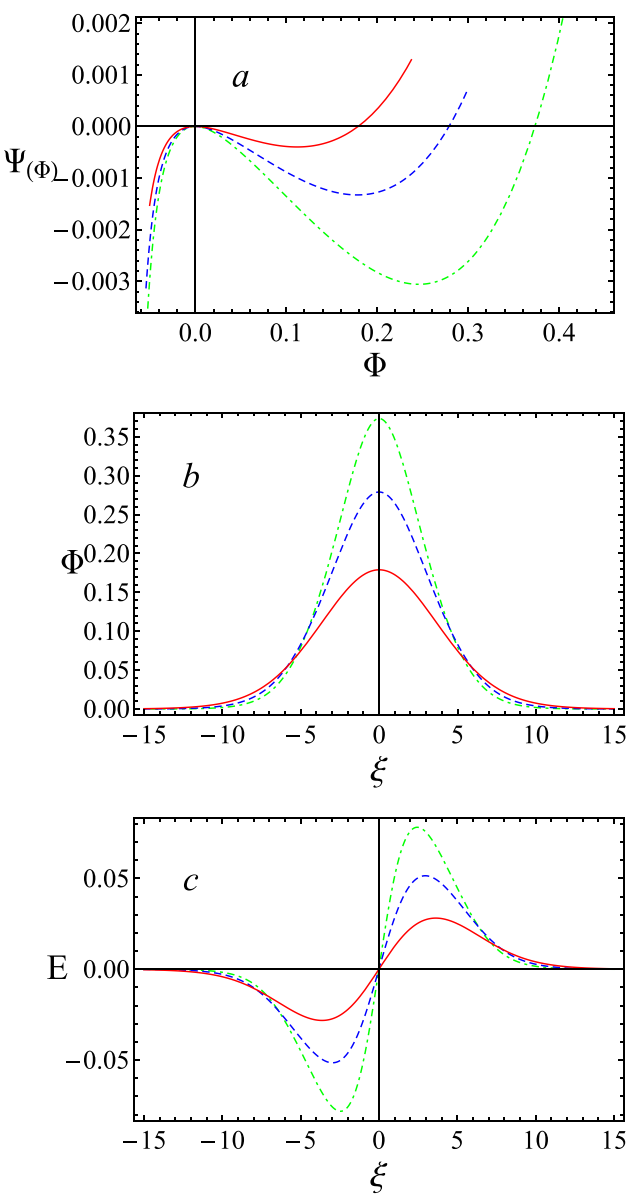

FIG. 5. (a) The pseudopotential $\Psi(\Phi)$ is depicted versus $\Phi$ for $\kappa=3$, $M=0.7, P_{\|}=0.03, P_{\perp}=0.01, \Omega=0.5$, and: $\alpha=0.7$ (dotted-dashed green curve), $\alpha=0.75$ (dashed blue curve), $\alpha=0.8$ (solid red curve). (b) The corresponding electrostatic potential and (c) the resulting electric field are shown.

\section{CONCLUSIONS}

We have investigated the nonlinear properties of arbitrary amplitude ion-acoustic excitations (solitary waves), propagating in a magnetized plasma characterized by anisotropic ions and by kappa-distributed electrons. In the linear regime, we have obtained two modes, corresponding to the magnetized ion-acoustic and to the ion-cyclotron modes, whose characteristics depend on the (superthermal) electron distribution and on the pressure anisotropy of the ions. In the nonlinear regime, the properties of arbitrary amplitude obliquely propagating ion-acoustic solitary waves were studied via a Sagdeev pseudopotential approach, based on a pseudo-mechanical energy balance analogy.

A parametric analysis has led to a number of qualitative conclusions, which we summarize in the following. First of all, the soliton existence region, in terms of the permitted Mach number values, is delimited by two extreme values, i.e., in the interval $\left(M_{1}, M_{2}\right.$. This interval reduces in size with smaller values of $\kappa$ (i.e., for a stronger deviation from the Maxwellian state), while a constant (saturation) value is attained at higher $\kappa$ (i.e., for a given value of the directional cosine, expressed via the parameter $\alpha$ in our model). Similarly, the soliton Mach number range decreases with stronger obliqueness (deviation from the direction parallel to
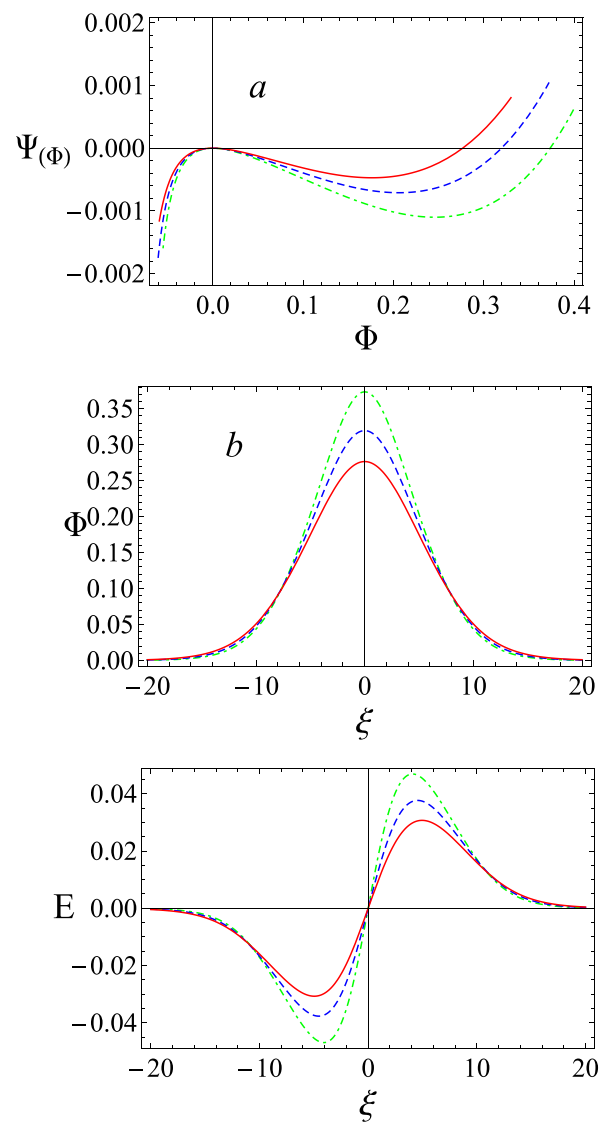

FIG. 6. (a) The pseudopotential $\Psi(\Phi)$ is depicted versus $\Phi$ for $\kappa=3$, $M=0.7, P_{\perp}=0.01, \alpha=0.7, \quad \Omega=0.3, \quad$ and: $P_{\|}=0.03 \quad$ (dotted-dashed green curve), $P_{\|}=0.06$ (dashed blue curve); $P_{\|}=0.09$ (solid red curve). (b) The corresponding electrostatic potential and (c) the resulting electric field are shown.

the ambient magnetic field), while at the same time it shifts towards higher values.

Both lower and upper Mach number critical values increase with the parallel (ion) pressure $P_{\|}$, for a specific value of $P_{\perp}$. The perpendicular (ion) pressure $P_{\perp}$ only affects the upper Mach number, while the lower Mach number (threshold) $M_{1}$ is essentially independent of $P_{\perp}$.

We have observed that by decreasing the superthermality parameter $\kappa$ (i.e., for stronger superthermality, i.e., a stronger deviation from the Maxwellian), the pulse amplitude increases significantly, and hence, solitary waves are more localized and steeper, due to energetic electrons.

It was also found that, considering faster pulses, i.e., as $M$ increases, both the amplitude and depth of the pseudopotential increase well; therefore, the structures with larger $M$ (within the interval $M_{1}<M<M_{2}$ ) are expected to be taller and sharper (narrower).

The amplitude of the soliton turns out to be independent from the magnetic field (strength), expressed via $\Omega$, which nonetheless affects (increases) the depth of the Sagdeev potential well, which increases with larger $\Omega$. The potential pulse's shape will therefore depend on the magnetic field (wider for stronger $\Omega$ ), but not its amplitude.

Finally, the characteristics of electrostatic solitary waves are more sensitive to the parallel (ion) pressure component $P_{\|}$than to its perpendicular counterpart $P_{\perp}$. 

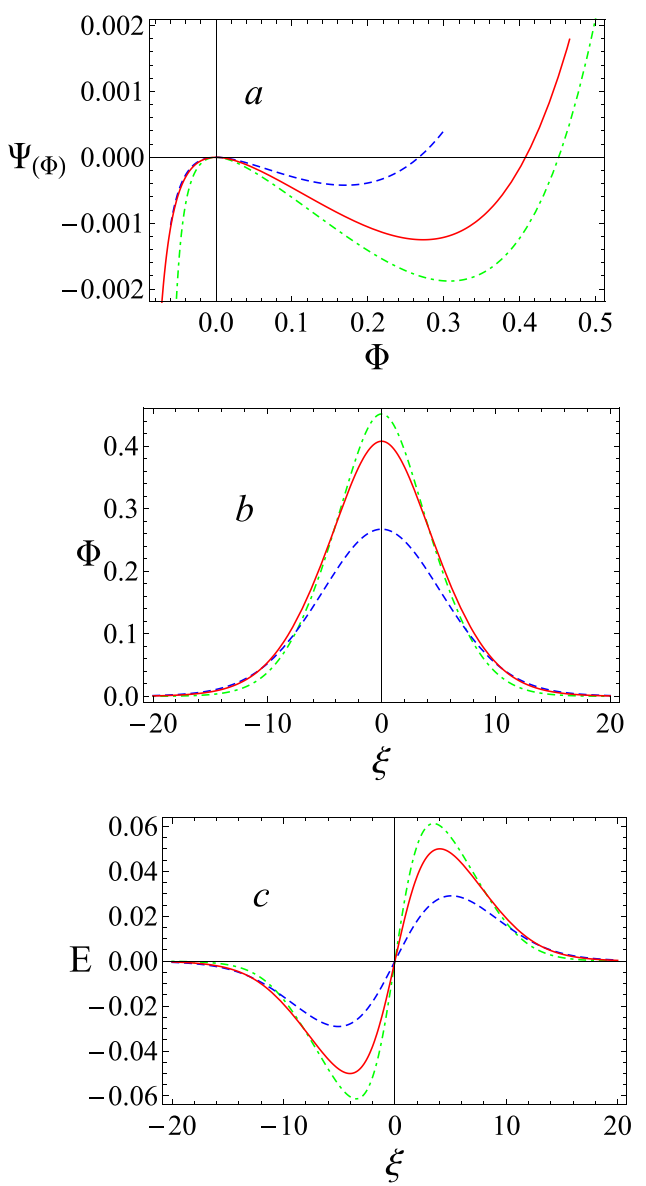

FIG. 7. (a) The pseudopotential $\Psi(\Phi)$ is depicted versus $\Phi$ for $\kappa=3$, $M=0.7, \alpha=0.7, \Omega=0.3$, and: $P_{\|}=P_{\perp}=0$ (dotted-dashed green curve); $P_{\|}=0.1$ and $P_{\perp}=0.02$ (dashed blue curve); $P_{\|}=0.02$ and $P_{\perp}=0.1$ (solid red curve). (b) The corresponding electrostatic potential and (c) the resulting electric field are shown.

It may be added, for rigor, that we have not considered the actual mechanism of soliton generation, but rather, we have focused on the conditions for their existence instead. It is a standard working hypothesis that nonlinear modes (such as pulse solitons, here) occurring in integrable media represent in some sense "normal modes" for the given physical system. If these may exist in certain physical conditions (hence our interest in their conditions for existence), then an amount of energy launched in the plasma, in the form, e.g., of a localized lump of electromagnetic energy, or a local disturbance of the electrostatic potential, will evolve into a series of solitons. This scenario, however hypothetical, is corroborated by abundant observations in Space plasmas.

It may be pointed out that we have employed the same physical (plasma-fluid) model in our earlier investigation of electrostatic solitary waves in Ref. 26. The methodology adopted in that study is distinct from the pseudopotential analysis followed herein: As a matter of fact, the ZakharovKuznetsov (ZK) perturbative approach employed in Ref. 26 is only valid in the small-amplitude approximation, and for weakly superacoustic pulses. The tedious Sagdeev-type analysis adopted here relies on the neutrality hypothesis, but is otherwise unrestricted in (arbitrary) pulse amplitude and in the range of values for the velocity. In this sense, the work at hand extends and generalizes the results of Ref. 26.

Our results should provide a good qualitative description of the dynamics of solitary waves, as these are observed in various space and astrophysical environments characterized by strong magnetic fields. This is particularly relevant in the magnetosphere and near Earth magnetosheath, ${ }^{17,18}$ where a non-thermal (energetic) electron distribution and ion pressure anisotropy can simultaneously occur. ${ }^{19-21}$

\section{APPENDIX: DERIVATION OF EQS. (27)-(29)}

We present here the most important steps in deriving the Sagdeev-type potential equation. Substituting Eq. (24) with Equations (22) and (23), we have obtained

$$
\begin{gathered}
-\frac{M}{n_{i}} \frac{d v_{i y}}{d \xi}+\beta \frac{d \Phi}{d \xi}-\Omega v_{i z}+\beta P_{\perp} \frac{1}{n_{i}} \frac{d n_{i}}{d \xi}=0, \\
-\frac{M}{n_{i}} \frac{d v_{i z}}{d \xi}+\Omega v_{i y}=0 .
\end{gathered}
$$

Using the value of $v_{i y}$ from Eq. (26) in Eq. (A2), one obtains

$$
\begin{aligned}
\frac{d v_{i z}}{d \xi}= & \Omega\left[\frac{n_{i}}{\beta}\left(1-\frac{1}{n_{i}}\right)-\frac{\alpha^{2}}{M^{2} \beta}\right. \\
& \left.\times\left\{-n_{i}+n_{i} \int n_{i} d \Phi+\frac{1}{3} P_{\|} n_{i}\left(n_{i}^{3}-1\right)\right\}\right] .
\end{aligned}
$$

Differentiating Eq. (A1) w.r.t. $\xi$ and using the values $v_{i y}$ and $d v_{i z} / d \xi$, we get

$$
\begin{aligned}
& \left(\frac{3 M^{2}+\alpha^{2} P_{\|} n_{i}^{4}-\beta^{2} P_{\perp} n_{i}^{2}}{n_{i}^{4}}\right)\left(\frac{d n_{i}}{d \xi}\right)^{2} \\
& -\left(\frac{M^{2}-\alpha^{2} P_{\|} n_{i}^{4}-\beta^{2} P_{\perp} n_{i}^{2}}{n_{i}^{3}}\right)\left(\frac{d^{2} n_{i}}{d \xi^{2}}\right)+\frac{d^{2} \Phi}{d \xi^{2}}=F(\Phi) .
\end{aligned}
$$

Using Eq. (5), one can express the above Equation (A4) in terms of the electrostatic potential $\Phi$

$$
\begin{aligned}
& \frac{M^{2}(\kappa-1 / 2)}{(\kappa-3 / 2)^{2}}(2 \kappa-2)\left(1-\frac{\Phi}{\kappa-3 / 2}\right)^{2 \kappa-3}\left(\frac{d \Phi}{d \xi}\right)^{2} \\
& -\frac{M^{2}(\kappa-1 / 2)}{(\kappa-3 / 2)}\left(1-\frac{\Phi}{\kappa-3 / 2}\right)^{2 \kappa-2}\left(\frac{d^{2} \Phi}{d \xi^{2}}\right) \\
& +\frac{\alpha^{2} P_{\|}(\kappa-1 / 2)}{(\kappa-3 / 2)^{2}}(2 \kappa)\left(1-\frac{\Phi}{\kappa-3 / 2}\right)^{-2 \kappa-1}\left(\frac{d \Phi}{d \xi}\right)^{2} \\
& +\frac{\alpha^{2} P_{\|}(\kappa-1 / 2)}{(\kappa-3 / 2)}\left(1-\frac{\Phi}{\kappa-3 / 2}\right)^{-2 \kappa}\left(\frac{d^{2} \Phi}{d \xi^{2}}\right) \\
& +\frac{P_{\perp} \beta^{2}(\kappa-1 / 2)}{(\kappa-3 / 2)^{2}}\left(1-\frac{\Phi}{\kappa-3 / 2}\right)^{-2}\left(\frac{d \Phi}{d \xi}\right)^{2} \\
& +\frac{P_{\perp} \beta^{2}(\kappa-1 / 2)}{(\kappa-3 / 2)}\left(1-\frac{\Phi}{\kappa-3 / 2}\right)^{-1}\left(\frac{d^{2} \Phi}{d \xi^{2}}\right)+\frac{d^{2} \Phi}{d \xi^{2}}=F(\Phi) .
\end{aligned}
$$


Differentiating twice with respect to $\xi$, Equation (A5) above reduces to

$$
\begin{aligned}
& \frac{M^{2}}{2} \frac{d^{2}}{d \xi^{2}}\left(1-\frac{\Phi}{\kappa-3 / 2}\right)^{2 \kappa-1}+\frac{\alpha^{2} P_{\|}}{2} \frac{d^{2}}{d \xi^{2}}\left(1-\frac{\Phi}{\kappa-3 / 2}\right)^{-2 \kappa+1} \\
& +\beta^{2} P_{\perp}(-\kappa+1 / 2) \frac{d^{2}}{d \xi^{2}}\left[\ln \left(1-\frac{\Phi}{\kappa-3 / 2}\right)\right]+\left(\frac{d^{2} \Phi}{d \xi^{2}}\right)=F(\Phi) \\
& \Rightarrow \frac{d^{2} S}{d \xi^{2}}=F(\Phi)
\end{aligned}
$$

where

$$
\begin{aligned}
S= & {\left[\Phi+\frac{M^{2}}{2}\left(1-\frac{\Phi}{\kappa-3 / 2}\right)^{2 \kappa-1}+\frac{\alpha^{2} P_{\|}}{2}\left(1-\frac{\Phi}{\kappa-3 / 2}\right)^{-2 \kappa+1}\right.} \\
& \left.+\beta^{2} P_{\perp} \ln \left(1-\frac{\Phi}{\kappa-3 / 2}\right)^{-\kappa+1 / 2}\right] .
\end{aligned}
$$

Differentiating $S$ with respect to $\xi$ and squaring, we write

$$
\left(\frac{d S}{d \xi}\right)^{2}=[G(\Phi)]^{2}\left(\frac{d \Phi}{d \xi}\right)^{2}
$$

where

$$
\begin{aligned}
G(\Phi)= & {\left[1-M^{2}\left(\frac{\kappa-1 / 2}{\kappa-3 / 2}\right)\left(1-\frac{\Phi}{\kappa-3 / 2}\right)^{2 \kappa-2}\right.} \\
& +\alpha^{2} P_{\|}\left(\frac{\kappa-1 / 2}{\kappa-3 / 2}\right)\left(1-\frac{\Phi}{\kappa-3 / 2}\right)^{-2 \kappa} \\
& \left.+\beta^{2} P_{\perp}\left(\frac{\kappa-1 / 2}{\kappa-3 / 2}\right)\left(1-\frac{\Phi}{\kappa-3 / 2}\right)^{-1}\right]
\end{aligned}
$$

Multiplying both sides of Eq. (A7) by $\frac{d S}{d \xi}$, we obtain

$$
\frac{d}{d \xi}\left[\frac{1}{2}\left(G(\Phi) \frac{d \Phi}{d \xi}\right)^{2}\right]=F(\Phi) G(\Phi) \frac{d \Phi}{d \xi} .
$$

Integrating the latter equation under the boundary conditions $\Phi \rightarrow 0$ and $\frac{d \Phi}{d \xi} \rightarrow 0$ at $\xi \rightarrow \pm \infty$, we obtain Equation (29).

${ }^{1}$ V. M. Vasyliunas, J. Geophys. Res. 73, 2839, doi:10.1029/ JA073i009p02839 (1968).

${ }^{2}$ L. D. De Feiter and C. De Jager, Sol. Phys. 28, 183 (1973).

${ }^{3}$ J. D. Scudder, E. C. Sittler, and H. S. Bridge, J. Geophys. Res. 86, 8157, doi:10.1029/JA086iA10p08157 (1981).

${ }^{4}$ M. P. Leubner, J. Geophys. Res. 87, 6335, doi:10.1029/JA087iA08p06335 (1982).

${ }^{5}$ M. A. Hellberg, R. L. Mace, T. K. Baluku, I. Kourakis, and N. S. Saini, Phys. Plasmas 16, 094701 (2009).
${ }^{6}$ G. Livadiotis and D. J. McComas, Space Sci. Rev. 175, 183 (2013).

${ }^{7}$ W. C. Feldman, R. C. Anderson, J. R. Asbridge, S. J. Bame, J. T. Gosling, and R. D. Zwickl, J. Geophys. Res. 87, 632, doi:10.1029/ JA087iA02p00632 (1982).

${ }^{8}$ V. Pierrard and J. F. Lemaire, J. Geophys. Res. 101, 7923, doi:10.1029/ 95JA03802 (1996).

${ }^{9}$ M. Maksimovic, V. Pierrard, and J. F. Lemaire, Astron. Astrophys. 324, 725 (1997)

${ }^{10}$ S. Magni, H. E. Roman, R. Barni, C. Riccardi, Th. Pierre, and D. Guyomarc'h, Phys. Rev. E 72, 026403 (2005).

${ }^{11}$ G. Livadiotis and D. J. McComas, J. Geophys. Res. 114, A11105, doi:10.1029/2009JA014352 (2009).

${ }^{12}$ I. Kourakis, S. Sultana, and M. A. Hellberg, Plasma Phys. Controlled Fusion 54, 124001 (2012).

${ }^{13}$ G. Williams and I. Kourakis, Plasma Phys. Controlled Fusion 55, 055005 (2013).

${ }^{14} \mathrm{~W}$. Baumjohann and R. A. Treumann, Basic Space Plasma Physics (Imperial College Press, London, 1997).

${ }^{15}$ G. F. Chew, M. L. Goldberger, and F. E. Low, Proc. R. Soc. London, Ser. A 236, 112 (1956).

${ }^{16}$ G. K. Parks, Physics of Space Plasmas (Perseus, USA, 1991).

${ }^{17}$ R. E. Denton, B. J. Anderson, S. P. Gary, and S. A. Fuselier, J. Geophys. Res. 99(A6), 11225, doi:10.1029/94JA00272 (1994).

${ }^{18}$ C. R. Choi, C.-Mo. Ryu, D. Y. Lee, N. C. Lee, and Y. H. Kim, Phys. Lett. A 364, 297 (2007).

${ }^{19}$ G. Noci, J. L. Kohl, and G. L. Withbroe, Astrophys. J. 315, 706 (1987).

${ }^{20}$ M. Pagel, Q. D. Atkinson, and A. Meade, Nature 449, 717 (2007).

${ }^{21}$ F. Stephan, C. H. Boulware, M. Krasilnikov, J. Bähr, G. Asova, A. Donat, U. Gensch, H. J. Grabosch, M. Hänel, L. Hakobyan et al., Phys. Rev. Spec. Top. Accel. Beams 13, 020704 (2010).

${ }^{22}$ A. Bret and C. Deutsch, Phys. Plasmas 13, 022110 (2006).

${ }^{23}$ A. Sid, A. Ghezal, A. Soudani, and M. Bekhouche, Plasma Fusion Res. 5, 7 (2010).

${ }^{24}$ J. Seough, P. H. Yoon, K. Kim, and D. Lee, Phys. Rev. Lett. 110, 071103 (2013).

${ }^{25}$ S. Peter Gary and M. A. Lee, J. Geophys. Res. 99(A6), 11297, doi:10.1029/94JA00253 (1994).

${ }^{26}$ M. Adnan, G. Williams, A. Qamar, S. Mahmood, and I. Kourakis, Eur. Phys. J. D 68, 247 (2014).

${ }^{27}$ M. Gedalin, Phys. Rev. E 47, 4354 (1993).

${ }^{28}$ W. B. Gebretsadkan and G. L. Kalra, Phys. Rev. E 66, 057401 (2002).

${ }^{29}$ P. Chatterjee, T. Saha, and C.-M. Ryu, Phys. Plasmas 15, 123702 (2008).

${ }^{30}$ C. R. Choi, C. M. Ryu, N. C. Lee, and D. Y. Lee, Phys. Plasmas 12, 022304 (2005).

${ }^{31}$ C. R. Choi, C. M. Ryu, D. Y. Lee, and Y. Kim, Phys. Plasmas 12, 073301 (2005).

${ }^{32}$ G. Livadiotis, J. Geophys. Res.: Space Phys. 120, 1607, doi:10.1103/ PhysRevE.63.061105 (2015); 120, 880 (2015).

${ }^{33}$ S. Mahmood, A. Mushtaq, and H. Saleem, New J. Phys. 5, 28 (2003).

${ }^{34}$ S. Sultana, I. Kourakis, N. S. Saini, and M. A. Hellberg, Phys. Plasmas 17, 032310 (2010).

${ }^{35}$ S. V. Singh, S. Devanandhan, G. S. Lakhina, and R. Bharuthram, Phys. Plasmas 20, 012306 (2013).

${ }^{36}$ N. S. Saini, I. Kourakis, and M. A. Hellberg, Phys. Plasmas 16, 062903 (2009).

${ }^{37}$ F. Verheest, J. Phys. A: Math. Theor. 42, 285501 (2009).

${ }^{38}$ R. Z. Sagdeev, in Reviews of Plasma Physics, edited by M. A. Leontovich (Consultants Bureau, New York, 1966), Vol. 4, p. 52.

${ }^{39} \mathrm{~F}$. Verheest and M. A. Hellberg, "Electrostatic solitons and Sagdeev pseudopotentials in space plasmas: Review of recent advances," in Handbook of Solitons: Research, Technology and Applications, edited by S. P. Lang and S. H. Bedore (NOVA Science Publishers, 2009). 